\title{
Coherent Control of High Harmonic Generation via Dual-Gas Multijet Arrays
}

\author{
A. Willner, ${ }^{1,2}$ F. Tavella, ${ }^{2}$ M. Yeung, ${ }^{3}$ T. Dzelzainis, ${ }^{3}$ C. Kamperidis, ${ }^{4}$ M. Bakarezos, ${ }^{4}$ D. Adams, ${ }^{3}$ M. Schulz, ${ }^{1,5}$ \\ R. Riedel, ${ }^{2,5}$ M. C. Hoffmann, ${ }^{6}$ W. Hu, ${ }^{6}$ J. Rossbach, ${ }^{5}$ M. Drescher, ${ }^{5}$ N. A. Papadogiannis, ${ }^{4}$ M. Tatarakis, ${ }^{4}$ \\ B. Dromey, ${ }^{3}$ and M. Zepf ${ }^{3, *}$ \\ ${ }^{1}$ Deutsches Elektronen Synchrotron, Notkestrasse 85, 22607 Hamburg, Germany \\ ${ }^{2}$ Helmholtz-Institut Jena, Max-Wien-Platz 1, 07743 Jena, Germany \\ ${ }^{3}$ Queens University, University Road, Belfast BT7 1NN, United Kingdom \\ ${ }^{4}$ Centre for Plasma Physics and Lasers, TEI of Crete, Evangelou Daskalaki Street 1, 74100 Rethymno \\ and Romanou Street 3, 73133 Chania, Crete, Greece \\ ${ }^{5}$ University of Hamburg, Luruper Chaussee 149, 22603 Hamburg, Germany \\ ${ }^{6}$ Max Planck Research Department for Structural Dynamics, University of Hamburg, CFEL, Notkestrasse 85, \\ 22607 Hamburg, Germany \\ (Received 8 July 2011; published 19 October 2011)
}

\begin{abstract}
High harmonic generation $(\mathrm{HHG})$ is a central driver of the rapidly growing field of ultrafast science. We present a novel quasiphase-matching (QPM) concept with a dual-gas multijet target leading, for the first time, to remarkable phase control between multiple HHG sources $(>2)$ within the Rayleigh range. The alternating jet structure with driving and matching zones shows perfect coherent buildup for up to six QPM periods. Although not in the focus of the proof-of-principle studies presented here, we achieved competitive conversion efficiencies already in this early stage of development.
\end{abstract}

PACS numbers: 52.38. $-\mathrm{r}, 52.50 . \mathrm{Dg}, 52.77 .-\mathrm{j}$

Coherent extreme ultraviolet (XUV) pulses of attosecond to femtosecond duration generated by high harmonic generation $(\mathrm{HHG})$ in gases $[1,2]$ are central to applications in ultrafast science [3-6] and the external seeding of freeelectron lasers (FELs) [7]. In terms of enhancing the brightness of such pulses, achieving absolute and independent phase control between multiple harmonic generation zones represents a major advance for HHG sources. It will allow the coherent superposition of multiple sources created by the same laser-an approach commonly known as quasiphase matching (QPM) [8]. In the case of HHG, QPM is typically implemented by allowing the signal to build up over one coherence length $L_{c}=\pi / \Delta k$ (the HHG halfperiod) and subsequently suppressing HHG for another coherence length (the matching half-period) until the driving field and harmonic field are in phase again (here $\Delta k=$ $k_{q}-q k_{f}$ is the $k$-vector mismatch between the $q$ th harmonic and the fundamental). The effectiveness of QPM has been demonstrated in the past using capillaries to achieve a modulation of the laser intensity via periodic variation of the capillary diameter [8,9] or multimode beating in a capillary [10,11]. Other schemes have involved HHG from a capillary discharge [12] or the use of counterpropagating pulses [13]. Since the intensity of $N$ atoms emitting radiation coherently increases as $N^{2}$ under true phase matching conditions, the superposition of $N_{\text {QPM }}$ identical HHG sources will increase the intensity of the $q$ th harmonic as $I_{q} \propto\left(N_{\mathrm{QPM}}\right)^{2}$ under ideal conditions $\left(N_{\mathrm{QPM}}\right.$ is the number of QPM periods consisting of a HHG and a matching half-period). The usual QPM schemes, while elegant advances in their own right, fall short of this ideal scenario, because the source in the matching half-period is only weakly suppressed, the gas in the matching half-period adds appreciable absorption, and/or because the scheme does not allow the phase correction in the matching halfperiod to be freely adjusted. All this is the key to full control of attosecond pulse production and to achieve the full theoretical enhancement of $\left(N_{\mathrm{QPM}}\right)^{2}$.

In order to meet the requirements for fully controlled coherent buildup, we have developed a novel dual-gas QPM concept based on alternating a HHG generating medium with passive matching hydrogen zones [see Fig. 1(a)]. The only restriction is that the HHG medium must have a higher ionization potential than hydrogen. If this condition is fulfilled, hydrogen will be fully ionized at the rising edge of the pulse. Since completely ionized hydrogen cannot emit further harmonic photons it acts as a completely passive medium and therefore the hydrogen half-period only adds a phase dominated by the free electron dispersion such that

$$
\phi_{q} \propto q L_{M} n_{e} r_{e} \lambda,
$$

where $L_{M}$ is the length of the matching half-period, $n_{e}$ the electron density, $r_{e}$ the classical electron radius, and $\lambda$ the fundamental wavelength. In addition, neutral and depleted hydrogen have minimal absorption for all wavelengths of interest compared to HHG media available. For scenarios that aim to exploit the high peak power [14] or high average power of current-day lasers [15,16], dual-gas QPM also has the advantage that it is compatible with free-propagating laser geometries instead of capillaries. While capillary waveguides provide an elegant solution 
a)

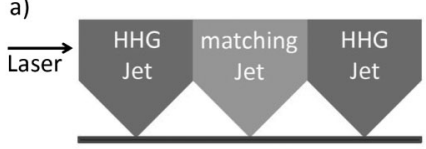

b)
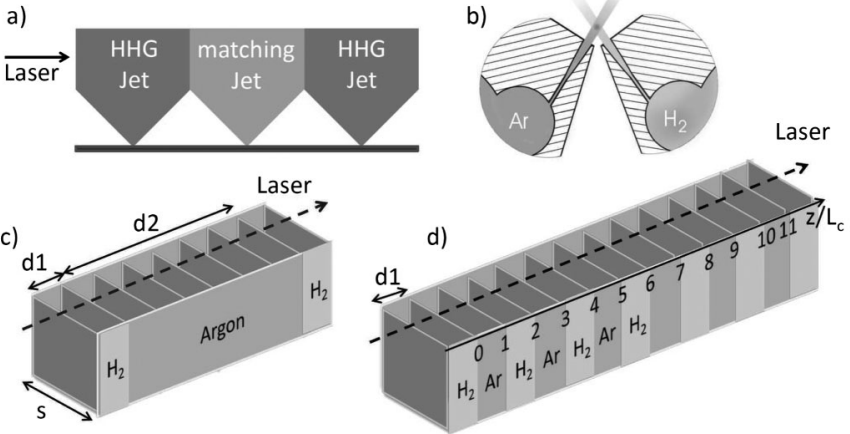

FIG. 1. (a) The dual-gas QPM principle. (b) Principle of the prototype target. It consists of two blocks with electroeroded Laval nozzles. The jets have a crossing angle of $75 \mathrm{deg}$. (c) Stacked foil target with 6 argon nozzles of $d 1=100 \mu \mathrm{m}$ and $s=1 \mathrm{~mm}$ used as a merged jet with two thin hydrogen jets for enclosing the argon density. The jets are separated by $20 \mu \mathrm{m}$ thin foils so that $d 2=700 \mu \mathrm{m}$. (d) QPM configuration with hydrogen jets separating the six argon jets. The hydrogen jets for density confinement remain.

to achieve near-uniform intensity over substantial interaction lengths, their lifetime can be limited by laser damage, especially under circumstances where the wall load is very high or when high average power systems being used are of particular interest for FEL seeding due to FEL pulse repetition rates of up to $1 \mathrm{MHz}$ [17]. Initial QPM schemes using free-propagating lasers and multijet arrays [18] free of the laser damage constraint were based on the variable spacing concept using the Gouy phase shift in vacuum as matching parameter $[19,20]$. This leads to large distances between the sources (on the order of millimeters) limiting the number of QPM periods $N_{\text {QPM }}$ that can be employed within the Rayleigh range of common laser systems. This restriction is most important when aiming to achieve QPM for very short wavelengths, where a small QPM period would allow for a large number of generation zones and hence the possibility of substantial signal enhancements. In particular, when trying to optimize critical HHG parameters like the cutoff energy, which requires a high intensity leading to a high ionization fraction, QPM with small QPM periods is the only way of enhancing the harmonic yield up to the theoretical limit.

A first proof-of-principle experiment was performed with a FemtoLasers amplifier system providing 30 fs pulses with $1 \mathrm{~mJ}$ pulse energy at a repetition rate of $1 \mathrm{kHz}$ and a central wavelength of $800 \mathrm{~nm}$. The laser radiation was focused by a parabolic mirror with an effective focal length of $f=150 \mathrm{~mm}$ leading to a measured focal spot of $40 \mu \mathrm{m}$ (FWHM). With a transmission efficiency of $86 \%$ a peak intensity of $8 \times 10^{14} \mathrm{~W} / \mathrm{cm}^{2}$ could be achieved. The jet array was positioned within the Rayleigh range of the focused laser beam. The jets were formed by electroeroded Laval-shaped nozzles with a diameter of $200 \mu \mathrm{m}$ at the nozzle exit. The separation of the nozzle centers was $430 \mu \mathrm{m}$. The nozzle blocks are constructed in a way that the arrays of argon and hydrogen had a crossing angle of 75 deg [see Fig. 1(b)], which leads to a crossing distance to the two nozzle entrances of about $150 \mu \mathrm{m}$. For this experiment, arrays with either two HHG jets and one matching jet or four HHG jets interspersed with three matching jets were used. The XUV radiation was detected using a flatfield XUV spectrometer and an ANDOR backthinned CCD camera. The IR light was attenuated with a $400 \mathrm{~nm}$ aluminum filter.

Figures 2(a) and 2(b) show the effect of hydrogen in an array of two argon jets separated by one hydrogen jet. The solid black curve represents the spectrum recorded with an argon backing pressure of 2 bar and a hydrogen pressure of 0.9 bar. Increasing the hydrogen backing pressure to 2.35 bar leads to the shaded gray spectrum. It is clear that hydrogen has an order-dependent effect on the spectrum such that harmonic orders beyond $q=27$ harmonic are enhanced whereas a decrease in harmonic yield can be observed for orders lower than the $q=25$ by changing the hydrogen pressure from 0.9 to 2.35 bar [see also Fig. 2(c)]. Such constructive or destructive interference between two sources is a signature of a QPM setup where an orderdependent phase $\phi_{q}$ is added between two sources. Comparing the two-source array with an array with four sources and three matching jets, an enhancement factor of $(4-2)^{2}=4$ is expected for perfect coherent buildup of the harmonic yield. To show this effect the two- and the

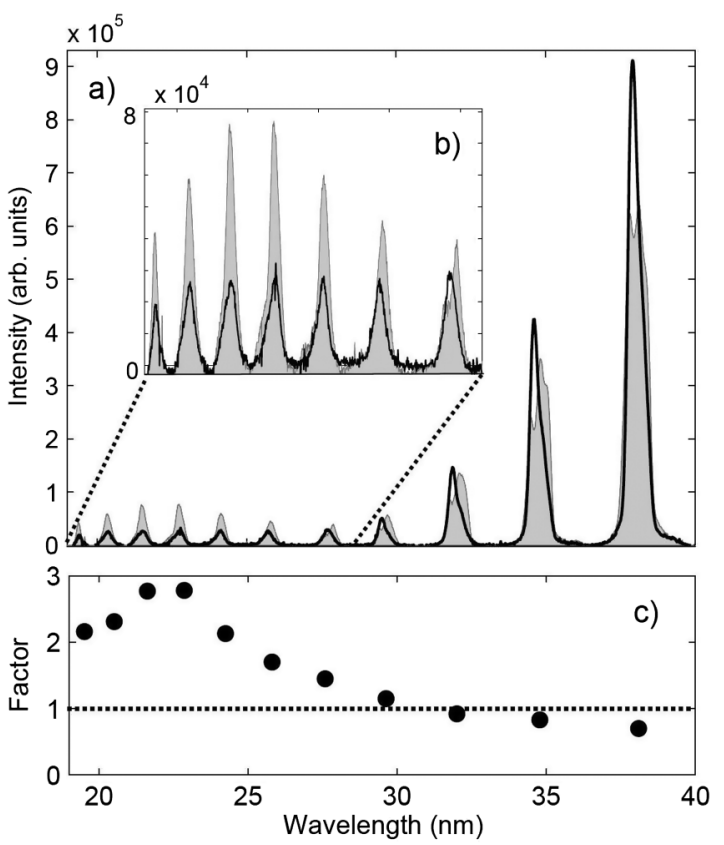

FIG. 2. (a) Selective enhancement due to QPM in the two nozzle array for two distinct values of hydrogen pressure (black solid, 0.9 bar of hydrogen; shaded gray, 2.35 bar). (b) Zoomed area of the harmonics 29-41. (c) Order-dependent enhancement due to the phase added by hydrogen. The argon backing pressure is 2 bar for all curves. 
four-source arrays were tuned to best phase matching with hydrogen for the 41st harmonic $(19.5 \mathrm{~nm})$. The argon backing pressure was kept constant at 2 bar where the harmonic signal from the argon emitters without any hydrogen showed the first rollover. Figure 3(a) shows the hydrogen tuning curve, and a maximum harmonic yield can be found at 1 bar hydrogen pressure. An enhancement factor of $3.9 \pm 0.2$ between two and four sources is clearly visible. Spectrally a peak-to-peak comparison of the 41 st harmonic should also lead to an enhancement factor of 4 with 1 bar hydrogen and 2 bar argon. In Fig. 3(b) a peak-topeak factor of $3.7 \pm 0.2$ can be observed, which is well within theoretical expectations. Hence, we can find a factor of $\approx 4$ in the spectrum and in the integrated signal of this harmonic. The splitting and blueshift in Fig. 3(b) is due to the longer path length for the four-source array and is caused by ionization blueshifting and quantum-path interference [21,22], which will be the subject of a further, more detailed publication.

As a consequence, the four-jet array is expected to give $16 \times$ the yield of a single jet under the same conditions. An elegant and simple method for directly observing the full enhancement factor due to pure QPM in multijet configurations was introduced by Seres et al. [20]. The theory makes the key simplification that the single-atom emission amplitude is the same for all atomic emitters. In addition, a dispersion dominated by free electrons is assumed leading to a linear dependence of the phase mismatch on the particle density of free electrons. The harmonic yield is then given by

$$
I_{q} \approx \frac{1-(-1)^{N_{\mathrm{QPM}}} \cos \left(\pi N_{n}\right)}{1+\cos \left(\pi / N_{\mathrm{QPM}} N_{n}\right)} \sin ^{2}\left(\frac{\pi N_{n}}{2 N_{\mathrm{QPM}}}\right) .
$$

$N_{\max , q}$ is the largest density of source gas particles for the single jet case $\left(N_{\mathrm{QPM}}=1\right)$ for which the intensity of the $q$ th harmonic monotonically grows and $N_{n}=N / N_{\max , q}$ is the normalized atomic density (with $N$ being the atomic
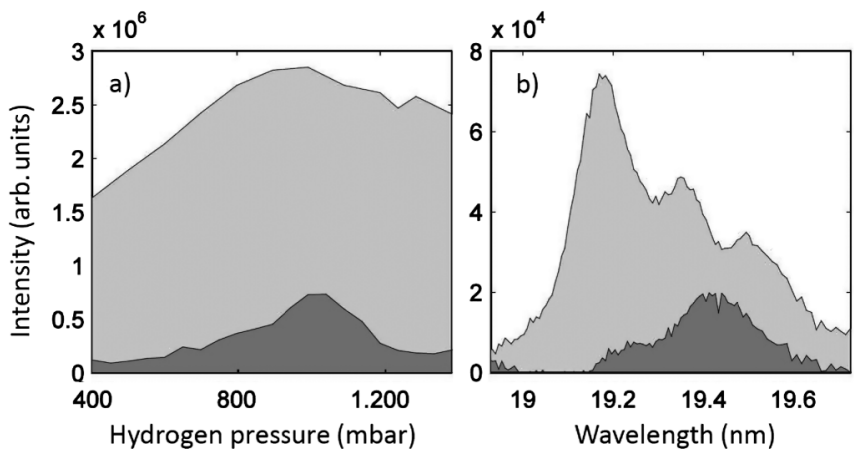

FIG. 3. Relative enhancement of the 41st harmonic $(19.5 \mathrm{~nm})$ between two sources (shaded dark gray) and four sources (shaded light gray). (a) Spectrally integrated tuning curve with variable hydrogen backing pressure and fixed argon pressure at 2 bar. (b) Peak-to-peak comparison in the angularly integrated spectrum at 1 bar hydrogen and 2 bar argon pressure. density). In this sense the normalized density is the point at which the length of the single or merged jet $L_{M}$ is just one coherence length $L_{c}$.

Best phase matching for a series of source jets (i.e., $N_{\text {QPM }}>1$ ) with an individual length of $L_{M} / N_{\text {QPM }}$ is then achieved by adjusting the pressure to a higher density of $N_{\mathrm{QPM}} N_{\text {max }, q}$, such that each individual jet in the series corresponds to one coherence length. This allows the quality of the QPM to be evaluated by comparing a configuration with $N_{\mathrm{QPM}}=1$ (i.e., a single jet without QPM) to a configuration with $N_{\mathrm{QPM}}>1$ while keeping the total length of the source gas (and therefore the geometry of the individual jets) constant.

To investigate the feasibility of the dual-gas QPM concept with higher source number, an experiment with six QPM periods was performed using a Red Dragon amplifier (KM-Labs) system provided $35 \mathrm{fs}$ pulses with up to $15 \mathrm{~mJ}$ pulse energy and a repetition rate of $1 \mathrm{kHz}$ at a central wavelength of $800 \mathrm{~nm}$. The laser radiation was focused by a lens with an effective focal length of $f=1000 \mathrm{~mm}$ leading to a measured focal spot of $70 \mu \mathrm{m}$ FWHM. At pulse energies of 4 and $2.8 \mathrm{~mJ}$ the peak intensity derived from focal measurements is $9 \times 10^{14}$ and $6 \times$ $10^{14} \mathrm{~W} / \mathrm{cm}^{2}$, respectively. The jet array was positioned within the Rayleigh range of the focused laser beam. To implement more sources, the advanced target design consists of multiple stacked foils with a rectangular nozzle orifice of $100 \mu \mathrm{m}[\times 1000 \mu \mathrm{m}$, transverse size; see Fig. 1(c) and 1(d)] instead of electroeroded nozzles, because the complicated prototype does not scale easily to larger jet numbers. In this configuration, the jets do not have any angle to each other. The backing pressures in the two arrays were controlled separately providing the same pressure within an array. Note that, in principle, our novel scheme allows the phase introduced by each matching halfperiod to be varied freely from one hydrogen zone to the next which will be implemented in a future version. In Fig. 4(a) two data sets are plotted versus argon backing pressure. At first a merged argon jet of $700 \mu \mathrm{m}$ length [consisting of six jets enclosed by two thin hydrogen jets of $100 \mu \mathrm{m}$ to avoid density spreading; see Fig. 1(c)] shows the characteristic phase oscillations with increasing argon pressure [see also Fig. 4(b)]. The nonzero signal in the minima with increasing pressure is due to off-axis phase matching [23], which is not considered in Eq. (2). The data points corresponds to the case $N_{\mathrm{QPM}}=1$ in Eq. (2) which is fitted to the data (darkly shaded) and allow the backing pressure corresponding to $N_{\max , q}$ to be estimated as 87.5 mbar. Finally, the QPM effect with hydrogen is shown in the data set with a $N_{\mathrm{QPM}}=6$ multijet array [consisting of the same six argon jets but now separated by hydrogen jets of $100 \mu \mathrm{m}$ length; see Fig. 1(d)]. By keeping the interaction length with argon constant, any enhancement occurring will be an effect of QPM. The hydrogen pressure was chosen to maximize the output at an argon backing 


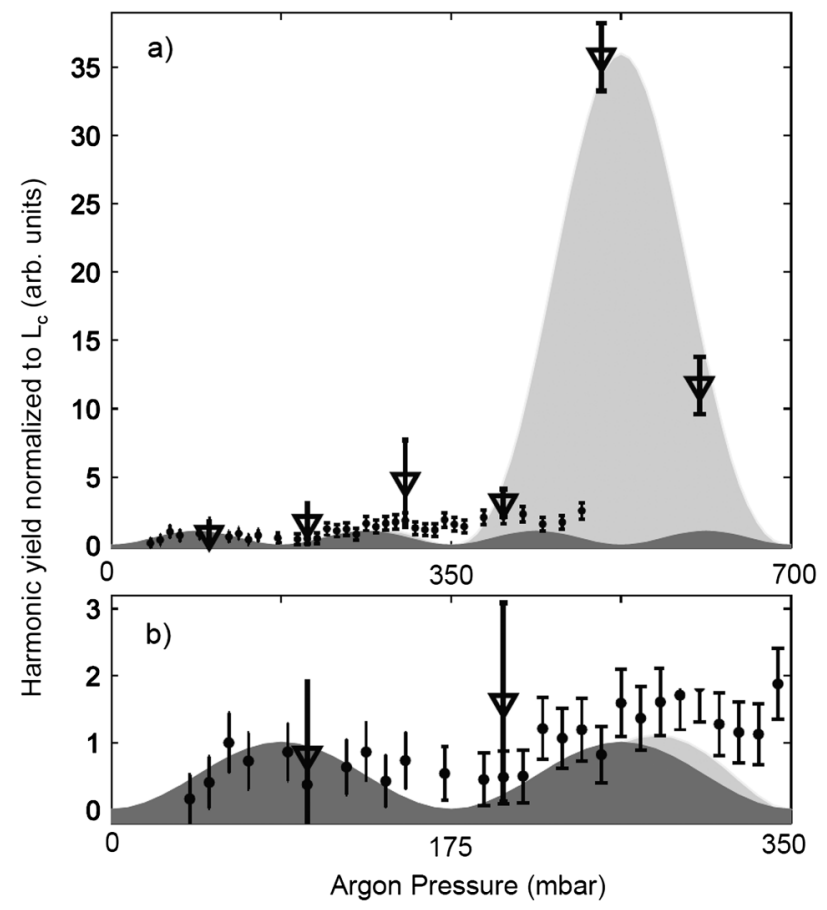

FIG. 4. (a) Comparison of the angularly and spectrally integrated harmonic yield for the 27th harmonic using a $700 \mu \mathrm{m}$ merged argon jet (circles) and a dual-gas six-sources jet (triangles) which has five hydrogen jets placed between the six argon jets. For comparison the theoretical curves are included for $N_{\mathrm{QPM}}=1$ (dark shaded) and $N_{\mathrm{QPM}}=6$ (light shaded). (b) Close-up of the merged-jet oscillations with increasing argon backing pressure.

pressure of $p=6 \times 87.5$ mbar $=525$ mbar (corresponding to a density of $N_{\mathrm{QPM}} N_{\mathrm{max}, q}$ ). As one can see, the measured data fit the theoretical expectation well for $N_{\text {QPM }}=6$ and we thus show, for the first time, an enhancement factor of 36 for a multijet array of six sources. For an intensity of $9 \times 10^{14} \mathrm{~W} / \mathrm{cm}^{2}$ the best conversion efficiencies achieved with the $N_{\mathrm{QPM}}=6$ array are $1.2 \times 10^{-5}, 9 \times$ $10^{-6}$, and $3 \times 10^{-6}$ for the 19th, 23rd, and 27th harmonic order, respectively, which is close to highest values reported for a $800 \mathrm{~nm}$ driver in the literature to date [24-26]. However, optimized conversion efficiency is not the primary aim of this novel study but rather will form the basis of future work.

To conclude, we have shown, for the first time, complete control of the laser-driven HHG process for multiple HHG sources by controlling the relative phase of the sources with matching zones consisting of fully ionized hydrogen gas inserted between the HHG zones. Controlling the process this way leads to quasiphase matching, which is confirmed by an enhancement of $\left(N_{\mathrm{QPM}}\right)^{2}$ (for up to $N_{\text {QPM }}=6$ ) in excellent agreement with theory. This new approach, not limited to any specific number of nozzles, represents a relatively simple tool for increasing the conversion efficiency to its theoretical limit. For short harmonic wavelengths, where current HHG efficiencies are substantially smaller than those that could be theoretically achieved, this method is anticipated to be particularly attractive. The low self-absorption at shorter wavelengths implies that jet arrays with $N_{\mathrm{QPM}}>10$ are possible with a decreased nozzle opening.

Ultimately from the current perspective the limit of the dual-gas QPM concept is only given by absorption and focusing geometry. To overcome the latter limit, laser systems with higher pulse energies can be used resulting in a focusing geometry with much larger confocal parameters while keeping the appropriate intensity for HHG. In addition, further optimization of the HHG process can be achieved by applying schemes such as shaped focal spots with flattop profiles. In principle, our scheme will extend to almost any medium as long as the ionization potential of the HHG medium exceeds that of hydrogen. These results are a major step towards the next generation of applications that require high peak and/or average power XUV sources.

We are grateful for the outstanding expertise in engineering of J. Gonschior (DESY Hamburg). We thank K. Schmid (MPQ Garching) for discussions concerning the supersonic micronozzles. We acknowledge the technical support at CPPL/TEI of Crete by S. Brezas. Thanks to A. Cavalleri for hosting the second experiment. A.W. acknowledges financial support from Graduiertenkolleg 1355 at the University of Hamburg.

*m.zepf@qub.ac.uk

[1] A. McPherson et al., J. Opt. Soc. Am. B 4, 595 (1987).

[2] X. F. Li et al., Phys. Rev. A 39, 5751 (1989).

[3] R.L. Sandberg et al., Phys. Rev. Lett. 99, 098103 (2007).

[4] E. Gagnon et al., Science 317, 1374 (2007).

[5] T. Haarlammert and H. Zacharias, Curr. Opin. Solid State Mater. Sci. 13, 13 (2009).

[6] M.E. Siemens et al., Appl. Phys. Lett. 94, 093103 (2009).

[7] G. Lambert et al., Nature Phys. 4, 296 (2008).

[8] A. Paul et al., Nature (London) 421, 51 (2003).

[9] I. P. Christov et al., Opt. Express 3, 360 (1998).

[10] M. Zepf et al., Phys. Rev. Lett. 99, 143901 (2007).

[11] B. Dromey et al., Opt. Express 15, 7894 (2007).

[12] D. M. Gaudiosi et al., Phys. Rev. Lett. 96, 203001 (2006).

[13] A. L. Lytle et al., Opt. Lett. 33, 174 (2008).

[14] C. J. Hooker et al., J. Phys. IV (France) 133, 673 (2006).

[15] F. Tavella et al., Opt. Express 18, 4689 (2010).

[16] J. Rothhardt et al., Opt. Express 18, 12719 (2010).

[17] B. Faatz et al., Nucl. Instrum. Methods Phys. Res., Sect. A 635, S2 (2010).

[18] M. Geissler, G. Tempea, and T. Brabec, Phys. Rev. A 62, 033817 (2000).

[19] A. Pirri, C. Corsi, and M. Bellini, Phys. Rev. A 78, 011801 (R) (2008). 
[20] J. Seres et al., Nature Phys. 3, 878 (2007).

[21] J.-H. Kim and Ch. H. Nam, Phys. Rev. A 65, 033801 (2002).

[22] X. He et al., Phys. Rev. A 79, 063829 (2009).

[23] P. Balcou et al., Phys. Rev. A 55, 3204 (1997).
[24] E. Constant et al., Phys. Rev. Lett. 82, 1668 (1999).

[25] J.F. Hergott et al., Phys. Rev. A 66, 021801(R) (2002).

[26] E. L. Falco-Filho et al., Appl. Phys. Lett. 97, 061107 (2010). 